\title{
Traumatic nondisplaced coronal suture fracture causing delayed intracranial hemorrhage in a pediatric patient
}

\author{
Tong Yang, MD, PhD \\ Sanford Brain and Spine Center, Sanford Children's Hospital, Fargo, North Dakota
}

\begin{abstract}
Skull fracture after a head injury is relatively common in children younger than 2 years of age. The author reports the case of a 14-month-old girl who sustained a unilateral nondisplaced coronal suture fracture from a fall. She developed delayed intracranial hemorrhage from an underlying dural tear and cortical vein injury. Although an isolated skull fracture in a pediatric trauma patient typically portends a benign clinical course and may not require that the patient be hospitalized, a nondisplaced fracture across the coronal suture can lead to dural tear and intracranial injuries. High vigilance is warranted when evaluating CT images around the suture lines and treating pediatric patients with fractures across the coronal suture.
\end{abstract}

https://thejns.org/doi/abs/10.3171/2017.3.PEDS1722

KEY WORDS coronal suture fracture; delayed intracranial hemorrhage; cortical venous injury; vascular disorders; trauma

$\mathrm{H}$ EAD injuries are relatively common in traumas involving children, especially in those younger than 2 years of age. Proposed guidelines ${ }^{21}$ based on available clinical data have been established to balance the benefit of effective treatment of head injury in young children with the risks of unnecessary radiation exposure and waste of medical resources. Here I report the case of an initially missed nondisplaced coronal suture fracture from a fall in a 14-month-old child, who subsequently developed a delayed intracranial hemorrhage needing surgical intervention.

\section{Case Report}

\section{Presentation}

The patient was a 14-month-old girl. Her mother was going upstairs to retrieve some clothing items when she heard a cry from the girl and screams from her older siblings. The mother found the girl somewhat lethargic and moaning by the side of the stairs. She presumed that the girl had followed her up the stairs and then fallen through the side of the stairs. The mother presumed that the child slid through between the railings on the side of the stairs and then fallen from a height of about 5 steps (approximately 5 feet) down to a hardwood floor. There was obvi- ous bruising on the girl's forehead. An ambulance arrived about 20 minutes after the accident. The emergency crew found the patient crying loudly and fighting restraints. She was brought to an outside hospital, arriving about an hour later. Head CT scanning performed about 2 hours after her fall was read as "normal" (Fig. 1). Although the mother felt that the child was still less active than her normal self, the patient was discharged about an hour later, around midnight, after the results of her examination were considered appropriate for her age. The mother was instructed to return if any concerning symptoms developed.

About 4 hours later (approximately 7 hours after her initial injury), the child woke up gagging and crying, and her mother also noted some twitching in her body. Another similar episode occurred again an hour later. She was then promptly brought back to the outside emergency room by the emergency crew. No obvious convulsive movement was noted when she was in the emergency room. A new CT scan of the brain showed a right frontal intraparenchymal hemorrhage (Fig. 2) with a nondisplaced fracture line crossing the coronal suture (missed by the providers on the previous scan). The patient was found to be listless with 4-mm sluggish pupils and a bigger scalp hematoma. She was not moving her left arm in response to intravenous sticks. She then became more tachycardic and diaphoretic. 

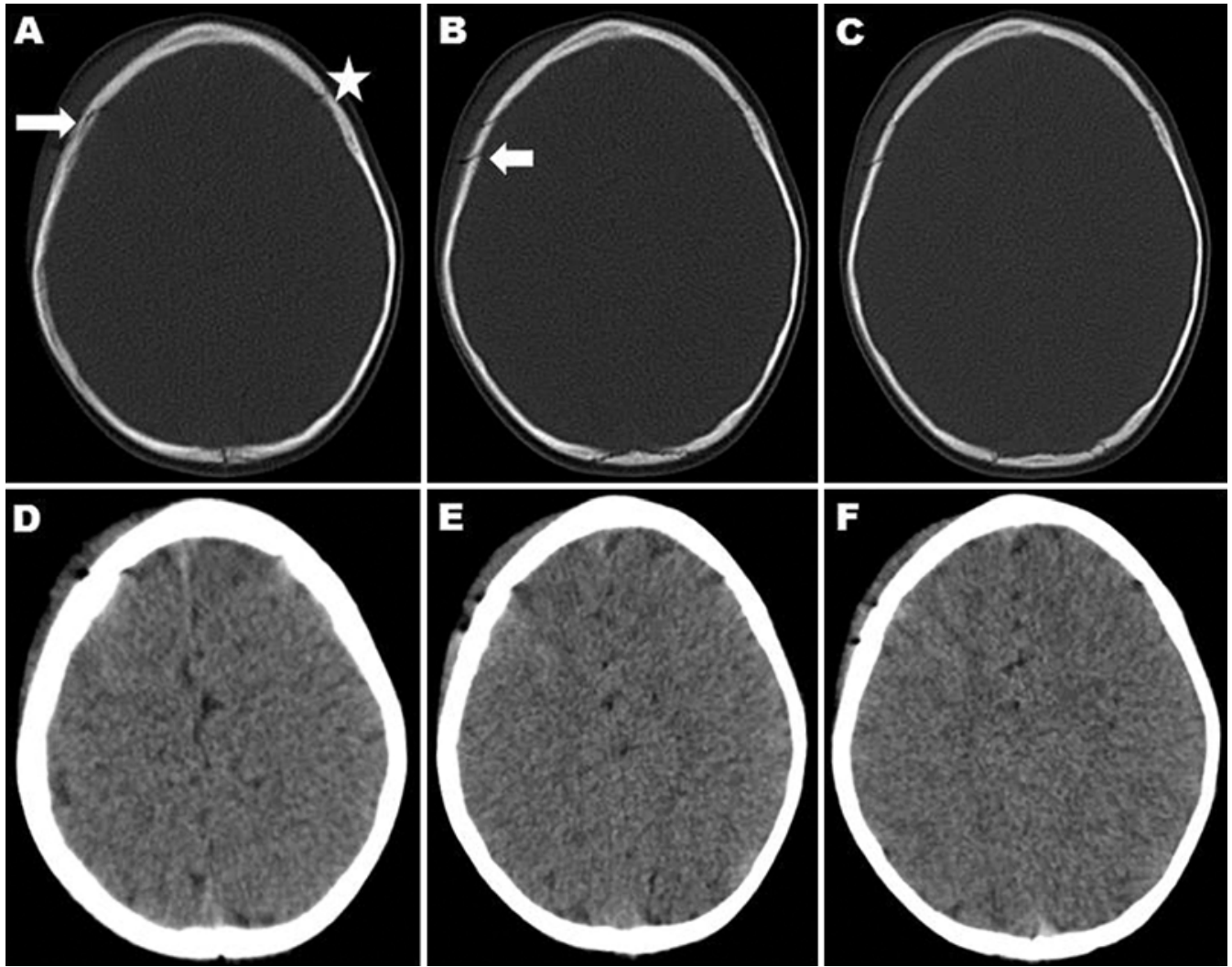

FIG. 1. Initial axial CT scans obtained about 2 hours after the injury. A: Bone window view showing the linear fracture line extending from the coronal suture on the right (arrow). It was missed and read as normal. Note the asymmetry compared with the intact coronal suture on the left (star). There is also obvious scalp swelling in the right frontal region. B: Bone window view a few cuts below the level in A, showing the continued nondisplaced fracture line (arrow). C: Bone window view a few cuts below the level in $B$, demonstrating the further extension of the fracture. D: Brain window view at the level corresponding to $A$, showing no obvious hemorrhage. E: Brain window view at the level corresponding to B, revealing no obvious hemorrhage. F: Brain window view at the level corresponding to $\mathrm{C}$, showing no obvious hemorrhage.

\section{Treatment}

The neurosurgeon on call examined the patient and found a subtle anisocoria and decreased responsiveness. Due to concern for brain herniation, the patient was given a dose of mannitol and taken to the operating room emergently for craniotomy. Intraoperatively, the linear fracture crossing the coronal suture was seen, and an associated dural tear was found. After the dura was further opened, a cortical vein was found to be bleeding briskly; the vein was associated with the tear site. The venous bleeding was stopped, and the hematoma was evacuated. The bone was replaced because the brain was found to be relaxed. The child was then transferred to the intensive care unit of our facility.

Blood samples were drawn for various laboratory evaluations. The hematocrit level was $25.1 \%$, platelet count $372 \mathrm{~K} / \mu \mathrm{l}$, prothrombin time 16.5 seconds, international normalized ratio 1.3 , activated partial thromboplastin time 30 seconds, and fibrinogen $244 \mathrm{mg} / \mathrm{dl}$.

MRI of the brain showed ischemic changes in the right frontal region (Fig. 3). Except for obvious postoperative changes on her head, there are no other bruises noted on her body. The child was extubated 4 days later. She was able to recognize her family and was able to talk. She had decreased movement on the left side but gradually re- gained function of the left leg with therapy. She started to show some movement of her left upper extremity but with notable weakness.

During her hospital stay, we also initiated nonaccidental trauma workup. Ophthalmological examination showed no retinal hemorrhage. Skeletal survey showed no other fractures except for the skull fracture and postcraniotomy changes.

\section{Outcome}

The child was discharged to home 12 days after admission to our pediatric intensive care unit. At the time of discharge, she was able to ambulate unassisted but still had decreased movement of the left arm. She was able to communicate verbally at her pre-event level. She was lost to follow-up because her parents are migrant workers and had been planning to move back to their home state prior to her accident.

\section{Discussion}

Brain injury is the most common cause of childhood death or permanent disability from trauma. ${ }^{10}$ Children younger than 2 years pose challenges in trauma evaluation and assessment due to their unique anatomical and de- 

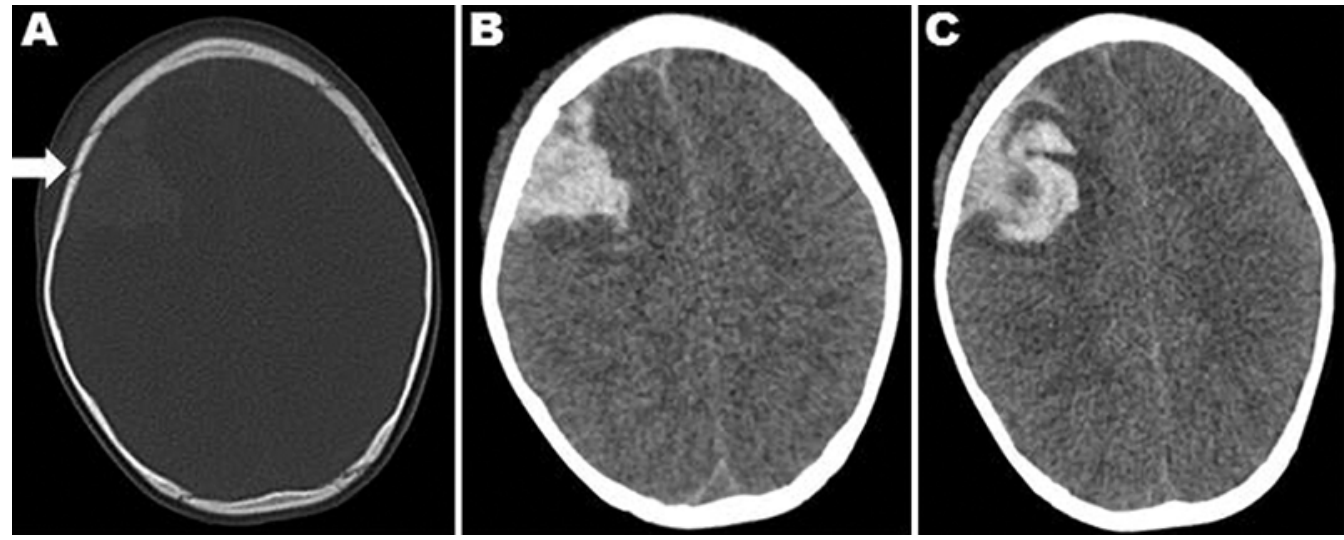

FIG. 2. Axial CT scans obtained about 7 hours postinjury after new neurological changes occurred. A: Bone window view at a level similar to Fig. 1B, showing the fracture line (arrow). A faint hyperdense intraparenchymal hemorrhage can be seen underlying the fracture. B: Brain window view at the level of Fig. $2 \mathrm{~A}$, demonstrating a wedge-shaped intraparenchymal hemorrhage with associated surrounding hypodense edema. C: Brain window view a few cuts lower than the level in Fig. 2B, again showing the intraparenchymal hemorrhage with associated edema.

velopmental characteristics. ${ }^{10,21}$ The increasing use of CT scans in pediatric trauma patients has generated concerns for risks of radiation-induced malignancy. ${ }^{6}$ In addition, based on the observation that most minor head injuries in young children portend a benign course, ${ }^{4,15}$ numerous studies have been carried out to identify clinical characteristics as indicators of underlying brain injury $y^{5,11,13,15}$ and the need to obtain CT scans..$^{2,8,15,18,20}$ Not a single clinical manifestation has been identified to be definitively associated with underlying intracranial injury, and in younger children, an intracranial injury may show no or only very subtle signs. Patients with a scalp hematoma and an associated skull fracture have been shown to have a higher chance of harboring an underlying intracranial injury; ;, $, 11,13,15$ however, young children with an isolated skull fracture have been found to have low probability of developing delayed intracranial hematoma ${ }^{16}$ or deterioration leading to the need for neurosurgical intervention. ${ }^{21}$ Reports looking at children with isolated skull fractures and normal examination results who are discharged from the emergency room ${ }^{19}$ or admitted for observations $\mathrm{s}^{12,19,21,22}$ have found no significant clinical deterioration or late complication. One proposal has been a period of observation in the emergency department (ED) without the need for hospitalization in asymptomatic patients harboring an isolated skull fracture. ${ }^{12,19,22}$

The various locations of skull fractures have not been systematically studied in relation to the development of underlying brain injuries. Most of the studies focused on fractures adjacent to a dural venous sinus and have shown that these types of fractures can potentially cause sinus thrombosis ${ }^{3,9}$ and hemorrhagic venous infarct, particularly if the superior sagittal sinus is involved. A few case reports on fractures involving the coronal sutures have reported a vertex epidural hematoma in patients with bilateral coronal suture diastasis after high-impact motor vehicle accidents, and with patients experiencing decreased neurological status. ${ }^{1,23}$

In the current case, the patient did fall from a height of more than 3 feet, and she was somewhat lethargic initially. She also had a scalp hematoma, which should have prompted the treating physicians to be more alert to look out for an underlying skull fracture. The fracture was nondisplaced, but there was an obvious asymmetry on the bone window between the bilateral coronal suture, which was unfortunately missed at the child's initial presentation. The first head CT scan was obtained about 2 hours after she fell, and she was observed for another hour after the CT scan before she was discharged, when she was reportedly "appropriate." This patient developed concerning symptoms about 4 hours after discharge (approximately 7 hours after her initial injury). Considering the typical turnover time in the ED, her deterioration was unlikely to have been picked up there unless she was admitted for observation as an inpatient. The guideline stated that "no sufficient data exist to comment on whether a child with a negative CT obtained a short time after the trauma requires a period of observation before discharge." ${ }^{21}$ Therefore the initial management by the local ED staff was not substandard, especially since the fracture was not identified by the reading radiologist. Even if the fracture were identified initially, without obvious intracranial hemorrhage on the initial CT, and with the child behaving appro-
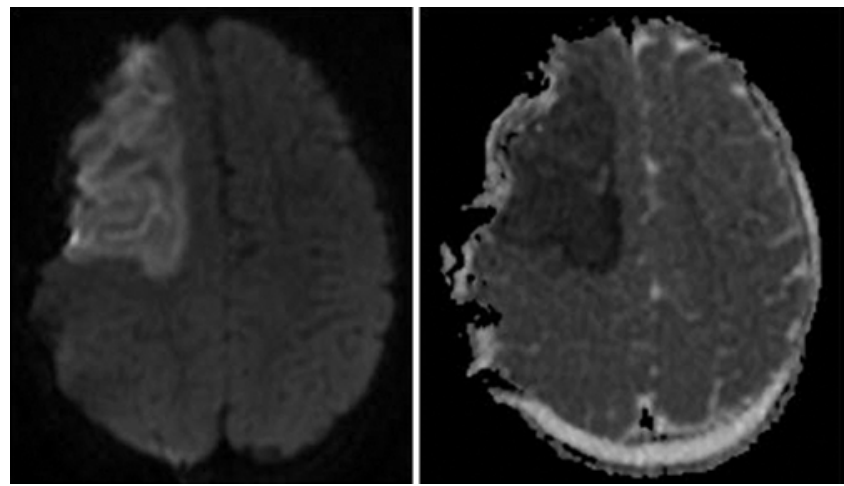

FIG. 3. MR images acquired after craniotomy. Left: Diffusion-weighted image showing a hyperintense signal in the right frontal region, consistent with ischemic changes. Right: Corresponding ADC (apparent diffusion coefficient) sequence showing hypointense signal change, consistent with its being ischemic in nature. 
priately, it would not have been negligent if she were not admitted. Her fracture did not cross the midline, which could cause potential superior sagittal sinus injury; however, it did extend to the coronal suture, where the dura can be tightly interdigitated with the suture line, and even a nondisplaced skull fracture can cause a dural tear and an underlying cortical vein injury if the vein happens to be in the vicinity.

The exact mechanism of the delayed hemorrhage was not obvious. Studies examining posttraumatic seizure occurrence in pediatric patients have found a low risk for mild injuries. ${ }^{14,17}$ Our patient could have had a seizure after she was discharged from the ED, but it is difficult to ascertain whether a seizure was the cause or result of the delayed hemorrhage. The patient did not show signs of more seizures after her treatment.

This case alerts us that a skull fracture involving the coronal suture, even when it does not cross the midline, should engender high clinical vigilance for potential underlying dura and cortical vein injury. A young patient with such injury should be observed closely for a longer period of time to avoid neurological deterioration and to receive timely neurosurgical intervention if indicated.

\section{Conclusions}

High vigilance is warranted when evaluating CT scans around the coronal suture lines in young head trauma patients, and a nondisplaced fracture across the coronal suture without crossing the midline can cause dural and cortical vein injury.

\section{References}

1. Arun P: A large vertex extradural hematoma due to traumatic bilateral coronal suture diastasis. Neurol India 59:935937, 2011

2. Beaudin M, Saint-Vil D, Ouimet A, Mercier C, Crevier L: Clinical algorithm and resource use in the management of children with minor head trauma. J Pediatr Surg 42:849852,2007

3. Benifla M, Yoel U, Melamed I, Merkin V, Cohen A, Shelef I: Dural sinus obstruction following head injury: a diagnostic and clinical study. J Neurosurg Pediatr 18:253-262, 2016

4. Berney J, Favier J, Froidevaux AC: Paediatric head trauma: influence of age and sex. I. Epidemiology. Childs Nerv Syst 10:509-516, 1994

5. Boran BO, Boran P, Barut N, Akgun C, Celikoglu E, Bozbuga $\mathrm{M}$ : Evaluation of mild head injury in a pediatric population. Pediatr Neurosurg 42:203-207, 2006

6. Brenner D, Elliston C, Hall E, Berdon W: Estimated risks of radiation-induced fatal cancer from pediatric CT. AJR Am J Roentgenol 176:289-296, 2001

7. Dacey RG Jr, Alves WM, Rimel RW, Winn HR, Jane JA: Neurosurgical complications after apparently minor head injury. Assessment of risk in a series of 610 patients. J Neurosurg 65:203-210, 1986

8. Dawson EC, Montgomery CP, Frim D, Koogler T: Is repeat head computed tomography necessary in children admitted with mild head injury and normal neurological exam? Pediatr Neurosurg 48:221-224, 2012

9. Delgado Almandoz JE, Kelly HR, Schaefer PW, Lev MH,
Gonzalez RG, Romero JM: Prevalence of traumatic dural venous sinus thrombosis in high-risk acute blunt head trauma patients evaluated with multidetector CT venography. Radiology 255:570-577, 2010

10. Gaynor BG, Zoeller GK, Ragheb J: Accidental head injuries in children, in Albright AL, Pollack IF, Adelson PD (eds): Principles and Practice of Pediatric Neurosurgery, ed 3. New York: Thieme, 2015, pp 701-705

11. Greenes DS, Schutzman SA: Clinical indicators of intracranial injury in head-injured infants. Pediatrics 104:861-867, 1999

12. Greenes DS, Schutzman SA: Infants with isolated skull fracture: what are their clinical characteristics, and do they require hospitalization? Ann Emerg Med 30:253-259, 1997

13. Greenes DS, Schutzman SA: Occult intracranial injury in infants. Ann Emerg Med 32:680-686, 1998

14. Huguenard AL, Miller BA, Sarda S, Capasse M, Reisner A, Chern JJ: Mild traumatic brain injury in children is associated with a low risk for posttraumatic seizures. J Neurosurg Pediatr 17:476-482, 2016

15. Kuppermann N, Holmes JF, Dayan PS, Hoyle JD Jr, Atabaki SM, Holubkov R, et al: Identification of children at very low risk of clinically-important brain injuries after head trauma: a prospective cohort study. Lancet 374:1160-1170, 2009

16. Mann KS, Chan KH, Yue CP: Skull fractures in children: their assessment in relation to developmental skull changes and acute intracranial hematomas. Childs Nerv Syst 2:258261, 1986

17. O’Neill BR, Handler MH, Tong S, Chapman KE: Incidence of seizures on continuous EEG monitoring following traumatic brain injury in children. J Neurosurg Pediatr 16:167176,2015

18. Quayle KS, Jaffe DM, Kuppermann N, Kaufman BA, Lee BCP, Park TS, et al: Diagnostic testing for acute head injury in children: when are head computed tomography and skull radiographs indicated? Pediatrics 99:E11, 1997

19. Rollins MD, Barnhart DC, Greenberg RA, Scaife ER, Holsti M, Meyers RL, et al: Neurologically intact children with an isolated skull fracture may be safely discharged after brief observation. J Pediatr Surg 46:1342-1346, 2011

20. Schunk JE, Rodgerson JD, Woodward GA: The utility of head computed tomographic scanning in pediatric patients with normal neurologic examination in the emergency department. Pediatr Emerg Care 12:160-165, 1996

21. Schutzman SA, Barnes P, Duhaime AC, Greenes D, Homer $\mathrm{C}$, Jaffe D, et al: Evaluation and management of children younger than two years old with apparently minor head trauma: proposed guidelines. Pediatrics 107:983-993, 2001

22. Vogelbaum MA, Kaufman BA, Park TS, Winthrop AL: Management of uncomplicated skull fractures in children: is hospital admission necessary? Pediatr Neurosurg 29:96-101, 1998

23. Wylen EL, Nanda A: Vertex epidural hematoma with coronal suture diastasis presenting with paraplegia. J Trauma 45:413-415, 1998

\section{Disclosures}

The author reports no conflict of interest concerning the materials or methods used in this study or the findings specified in this paper.

\section{Correspondence}

Tong Yang, Sanford Brain and Spine Center, Sanford Children's Hospital, 700 1st Ave. S, Fargo, ND 58103. email: tyangrun@ gmail.com. 